\title{
A modelling of AC voltage stabilizer based on a hybrid transformer with matrix converter
}

\author{
PAWEL SZCZEŚNIAK \\ Institute of Electrical Engineering, University of Zielona Góra \\ Licealna 9, 65-417 Zielona Góra, Poland \\ e-mail:P.Szczesniak@iee.uz.zgora.pl
}

(Received: 12.08.2016, revised: 27.01.2017)

\begin{abstract}
This article presents a study of an AC voltage stabilizer based on a three-phase hybrid transformer combined with a matrix converter. The proposed solution is used to control AC voltage amplitude and phase shift. By adjustment of these voltage parameters we can reduce the effects of overvoltage, voltage dips or lamp flicker. Such negative phenomena are very significant, particularly from the perspective of the final consumer and sensitive loads connected to the power network. Often the voltage in the power system can be adjusted using a mechanical or thyristor controlled regulator, which in a stepwise manner switches the taps of the electromagnetic transformer. The method for obtaining continuous control of the voltage magnitude and phase shift with the use of a conventional transformer with two output windings and a matrix converter is presented in this paper. The operating principles, mathematical model and properties of the proposed voltage stabilizers are discussed in this paper. The main part of the article will be devoted to the mathematical model which is based on an averaged equation. Computer simulation results are presented and compared with the results of a mathematical study.
\end{abstract}

Key words: AC voltage stabilizer, averaged model, hybrid transformer, matrix converter

\section{Introduction}

Electric Power Quality (EPQ) is a term that refers to the maintenance of a near sinusoidal waveform of power distribution bus voltages and currents at rated magnitude and frequency. Moreover, modern systems in industrial plants are based on high-tech equipment for increased productivity and this requires high quality electrical power. In addition, poor quality electricity can also cause damage to domestic electronic equipment, which more often than not now has miniature and large-scale integration of electronic circuits. Voltage sags are among the most significant power quality problems impacting on sensitive industrial and home equipment. Power quality standards define and classify voltage disturbances [1,2]. A large number of scientific papers are also dedicated to the issue of voltage disturbances in the power grid $[3,4]$. Power quality problems occur due to various types of electrical disturbances. Most of the EPQ disturbances impact on amplitude or frequency or on both. Voltage sags and interruptions are common phenomena resulting from network faults and dynamic load changes. 
Voltage sags are $\mathrm{rms} /$ magnitude reductions in the $\mathrm{AC}$ voltage for a duration ranging from a half-cycle to several dozen seconds.

In modern power systems, operators should prevent the causes and mitigate the effects of voltage distortion. The best possible solution for customers might be the installation of mitigation equipment for voltage disturbances. There are many solutions for power systems. The most common devices are tap changers (mechanical or solid-state) in electromagnetic transformers $[5,6]$. The second most common group consists of power electronic devices, which continuously stabilize the voltage at the point of common coupling (PCC) $[7,8]$. Such solutions are often called Custom Power Devices (CPD). There are two main methods for compensation of voltage-disturbances in power systems using power electronics devices: current oriented parallel devices (STATCOM-static synchronous compensator, SVC-static VAR compensator); and voltage oriented series devices (DVR-Dynamic Voltage Restorer) $[9,10]$. In this article only series compensation systems will be considered. The Dynamic Voltage Restorer (DVR) is one of the most efficient CPDs used to compensate fast voltage sags and overvoltages at PCC [9]. The DVR devices inject a voltage of the required magnitude, phase angle and frequency in series with the load. In this way the DVR maintains the set amplitude and waveform for the voltage at the load, even when the source voltage is distorted or unbalanced [7].

The concept of a hybrid transformer (HT) is defined as a combination of a classical electro-magnetic transformer with a power electronics converter. The literature describes a few concepts of HT with shunt connected converters, series connected converters and a combination of series and shunt connected converters [11-13]. The conception of the HT presented in this paper is based on a structure with a series connected converter. In the proposed HT the matrix converter (MC) is used $[14,15]$. Generally, the three-phase MC is a single-stage converter with an array of nine bi-directional switches. In order to ensure good performance, the MC should have a LC source filter to minimize the high frequency current distortion. The MC is a power device without a DC energy storage element. The DC energy storage element used in classical frequency converters with a voltage source inverter (VSI) is the main factor increasing size, weight and cost of the converter $[15,16]$. Furthermore, this energy storage in the form of electrolytic capacitors is the most frequently damaged element in operational service. Elimination of the DC energy magazine will increase the reliability of the proposed voltage compensator.

A preliminary study of the properties of the proposed MC based HT is presented in [12], where the idealized properties are shown. In addition, several similar solutions of AC voltage controllers for compensation of overvoltage and voltage sags (also based on the HT) are proposed in $[10,11,13,17-21]$. In the HT solution proposed in [11, 17] the PWM AC/AC matrix and matrix-reactance choppers are used [15]. The proposed choppers are employed for adjustment of the RMS value of the output voltages, excluding the possibility of output voltage phase shift adjustment. Using the HT with the matrix converter we obtain the possibility to control both the amplitude (or RMS value) and phase shift of the HT output voltages. This additional degree of regulation offers new possibilities for the application of the proposed solution, as previously shown in [12]. 
The main purpose of this paper is to describe the basic operations, based on the averaged mathematical model $[22,23]$ and simulation investigation, of the proposed HT with a space vector modulated (SVM) matrix converter. The simulation and theoretical results will be analysed and compared. In addition, the paper presents an application of the proposed HT to voltage sag compensation at the PCC in balanced and unbalanced conditions.

\section{Principles of the proposed solution}

As illustrated in Fig. 1, the proposed hybrid transformer consists of two main units: a three-phase matrix converter [14] and a conventional transformer. The transformer has two taps for each of the output phases. The first tap $\left(a_{1}, a_{2}, a_{3}\right.$-the names of first taps in each phase) is connected with the corresponding matrix converter input filter terminal $\left(L_{F}, C_{F}\right)$. Both the primary and secondary windings $a_{1}, a_{2}, a_{3}$ are in star-connections (Fig. 1). Subsequent seconddary windings $\left(b_{1}, b_{2}, b_{3}\right.$-the names of second taps in each phase) are connected in series with the corresponding MC output terminals. The voltage turn ratios of the windings $a_{1}, a_{2}, a_{3}$ and $b_{1}, b_{2}, b_{3}$ are equal to $n_{a}=4 / 3$ and $n_{b}=2 / 3$, respectively. The voltage gains have been adopted from the previous publications presenting HTs with AC choppers [11, 17]. It is possible to use other transformer turn ratios, as will be mentioned in the summary. The HT output voltage is the sum or result of the MC output voltage and output voltage of windings $b_{1}, b_{2}, b_{3}$. In order to extract the fundamental component of the MC output voltage, a low-pass $L_{L}, C_{L}$ output filter is employed.

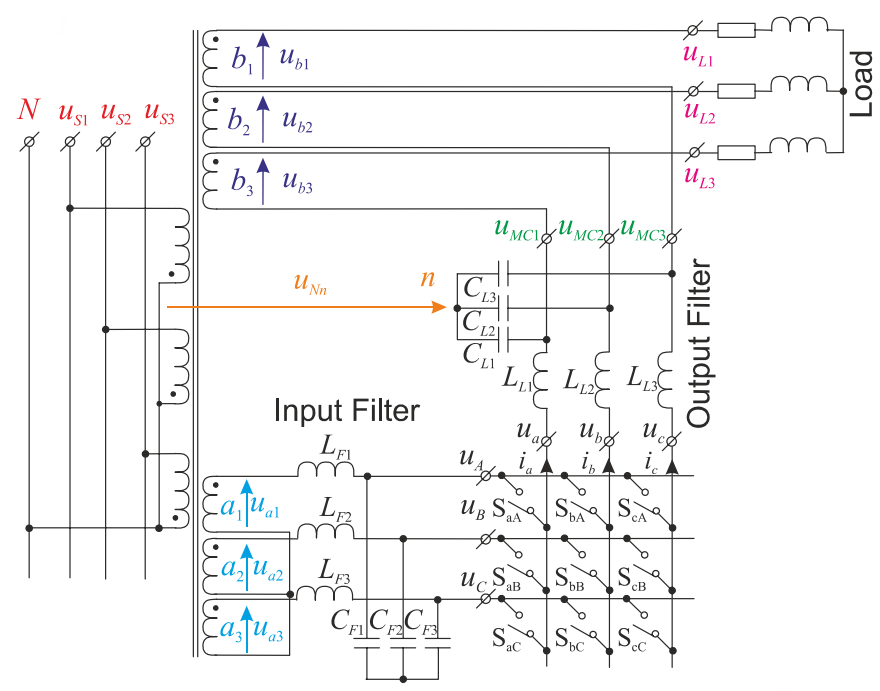

Fig. 1. Proposed hybrid transformer with MC and passive load

The MC output voltage is adjusted using voltage gain $q$ and the voltage phase shift $\varphi_{L}$. The maximum HT output voltage is achieved when the MC output voltages $\left(u_{M C 1}, u_{M C 2}, u_{M C 3}\right.$ are 
voltages at the output of $L_{L}, C_{L}$ filter) have the same phase as voltages $u_{b 1}, u_{b 2}, u_{b 3}$. If the source voltages are given as follows:

$$
\left[\begin{array}{c}
u_{S 1} \\
u_{S 2} \\
u_{S 3}
\end{array}\right]=\left[\begin{array}{c}
U_{S} \sin \left(\omega t+\varphi_{S}\right) \\
U_{S} \sin \left(\omega t+2 / 3 \pi+\varphi_{S}\right) \\
U_{S} \sin \left(\omega t-2 / 3 \pi+\varphi_{S}\right)
\end{array}\right]
$$

then the HT output voltages (for an ideal transformer without LC filters) are described by Eq. (2), where $U_{S}, U_{L}$ represent the amplitude of the input and HT output voltage, respectively, $\varphi_{S}$, $\varphi_{L}, \varphi$ represent the phase shift of the input voltage, MC output voltage and HT output voltage, respectively. To determine the exact HT voltage transmittance, it is necessary to describe a mathematical model that takes into account the parameters of components used in the LC filters and the MC modulation strategy.

$\left[\begin{array}{l}u_{L 1} \\ u_{L 2} \\ u_{L 3}\end{array}\right]=\left[\begin{array}{c}U_{S} \sin \left(\omega t+\varphi_{S}+\varphi_{L}\right) n_{a} q+U_{S} \sin \left(\omega t+\varphi_{S}\right) n_{b} \\ U_{S} \sin \left(\omega t+2 / 3 \pi+\varphi_{S}+\varphi_{L}\right) n_{a} q+U_{S} \sin \left(\omega t+2 / 3 \pi+\varphi_{S}\right) n_{b} \\ U_{S} \sin \left(\omega t-2 / 3 \pi+\varphi_{S}+\varphi_{L}\right) n_{a} q+U_{S} \sin \left(\omega t-2 / 3 \pi+\varphi_{S}\right) n_{b}\end{array}\right]=\left[\begin{array}{c}U_{L} \sin (\omega t+\varphi) \\ U_{L} \sin (\omega t+2 / 3 \pi+\varphi) \\ U_{L} \sin (\omega t-2 / 3 \pi+\varphi)\end{array}\right] .(2)$

The Space Vector Modulation (SVM) technique is the most frequently used modulation strategy for MC applications [14, 22]. The instantaneous space vector representation of load voltages $\left(\underline{u}_{O L}\right)$ and source currents $\left(\underline{i}_{S}\right)$ in the MC is presented as follows:

$\underline{u}_{O L}=\frac{2}{3}\left(u_{a b}+e^{-j 2 \pi / 3} u_{b c}+e^{j 2 \pi / 3} u_{c a}\right)=u_{O L} e^{j \alpha_{O L}(t)}, \underline{i}_{S}=\frac{2}{3}\left(i_{A}+e^{-j 2 \pi / 3} i_{B}+e^{j 2 \pi / 3} i_{C}\right)=i_{S} e^{j \beta_{1}(t)}$

The SVM method for the MC has been described many times in various publications (e.g., $[14,22])$. In this article there will be indicated only the basic formulas, useful for creating mathematical models. The reference space-vectors $\underline{u}_{O L}$ and $\underline{i}_{S}$ are constructed by selecting four basic "nonzero vectors" and one "zero vector", used during the appropriate time intervals within the switching period $T_{\text {Seq }}$. The required modulation duty cycles for the corresponding "nonzero vectors" are given by Eqs. (4) [22]:

$$
\begin{gathered}
d_{\mathrm{I}}=(-1)^{S_{0}+S_{i}+1} \frac{2 q \cos \left(\alpha_{0}-\pi / 3\right) \cos \left(\beta_{i}-\pi / 3\right)}{\sqrt{3} \cos \varphi_{i}}, d_{\mathrm{II}}=(-1)^{S_{0}+S_{i}} \frac{2 q \cos \left(\alpha_{0}-\pi / 3\right) \cos \left(\beta_{i}+\pi / 3\right)}{\sqrt{3} \cos \varphi_{i}}, \\
d_{\mathrm{III}}=(-1)^{S_{0}+S_{i}} \frac{2 q \cos \left(\alpha_{0}+\pi / 3\right) \cos \left(\beta_{i}-\pi / 3\right)}{\sqrt{3} \cos \varphi_{i}}, d_{\mathrm{IV}}=(-1)^{S_{0}+S_{i}+1} \frac{2 q \cos \left(\alpha_{0}+\pi / 3\right) \cos \left(\beta_{i}+\pi / 3\right)}{\sqrt{3} \cos \varphi_{i}},
\end{gathered}
$$

where: $\varphi_{i}$ is the input displacement angle, $\alpha_{0}$ and $\beta_{i}$ are the position angles of the output voltage and input current vectors, respectively, measured from the bisecting line of the corresponding sectors of the complex coordinate system, which are limited as follows: $-\pi / 6<\alpha_{0}<\pi / 6,-\pi / 6<\beta_{i}<\pi / 6$. The disadvantage of the MC is its limited voltage gain, which for the SVM is equal to $q_{\max }=\sqrt{3 / 2} \approx 0.866$. 
Knowing the maximum voltage gain of the $\mathrm{MC}$ and voltage turn ratios of the transformer windings $n_{a}$ and $n_{b}$, it is possible to determine the idealized expressions for the maximum and minimum HT output voltage [12]. The maximum HT output voltage for $n_{a}=4 / 3, n_{b}=2 / 3$ and an MC with SVM is defined by Eq. (5), whereas the minimum for the MC output voltage phase shift equals $180 \mathrm{deg}$, and is defined by Eq. (6). The relations between voltage phasors for $\underline{U}_{L}=\underline{U}_{L \max }, \underline{U}_{L}=\underline{U}_{L \min }$ and $\underline{U}_{L \max }<\underline{U}_{L}<\underline{U}_{L \min }$ for $\varphi_{L} \neq 0$, are shown in Fig. 2. The voltage adjustment range is limited by a circle, as shown in Fig. 2. It shows that in the proposed solution it is possible to compensate both voltage sags and overvoltage with the ability to control the phase shift of the output voltage.

$$
\begin{gathered}
U_{L \max }=U_{S} n_{a} q_{\max }+U_{S} n_{b}=U_{S} \frac{4}{3} \frac{\sqrt{3}}{2}+U_{s} \frac{2}{3} \approx 1.82 U_{S}, \\
U_{L \min }=-U_{S} \frac{4}{3} \frac{\sqrt{3}}{2}+U_{s} \frac{2}{3} \approx-0.49 U_{S} .
\end{gathered}
$$

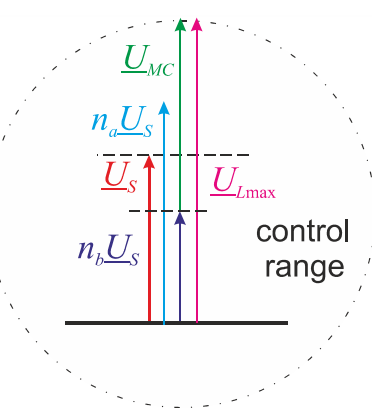

(a)

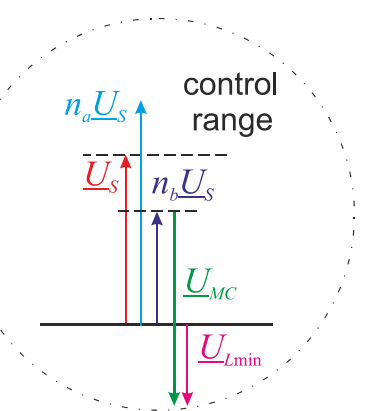

(b)

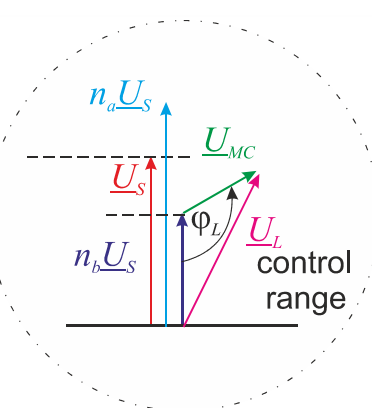

(c)

Fig. 2. HT voltage phasors for $n_{a}=4 / 3$ and $n_{b}=2 / 3$ and SVM: $U_{L}=U_{L \max }$ for $\varphi_{L}=0^{\circ}$ and $q_{\max }=0.866$ (a); $U_{L}=U_{L \min }$ for $\varphi_{L}=180^{\circ}$ and $q_{\max }=0.866(\mathrm{~b}) ; U_{L \max }<U_{L}<U_{L \min }$ for $\varphi_{L} \neq 0^{\circ}$ and $q_{\max }<0.866$ (c)

The mathematical description and characteristics presented above are defined in the idealized case, without influence of the LC filter and transformer parameters. For a precise depiction the properties of the analyzed system require a detailed mathematical model, which will be shown in the next section. The main problem in providing a detailed mathematical description of the analyzed HT is the inclusion of the modulation pattern of the MC switches. The proposed modelling process will be based on the state-space average model $[22,23]$.

\section{Modelling theory}

The presented mathematical model is based on the state-space equations for the averaged variables which are described by (7) [22].

$$
\frac{\mathrm{d} \overline{\boldsymbol{x}}}{\mathrm{d} t}=\boldsymbol{A}(d) \overline{\boldsymbol{x}}+\boldsymbol{B}(d),
$$


where: $\overline{\boldsymbol{x}}$ is the vector of the averaged state variables, $\boldsymbol{A}(d)$ is the averaged state matrix, $\boldsymbol{B}(d)$ is the averaged input matrix. This model is created by averaging of state variables in the sequence period $T_{S e q}$ for all switch combinations of the analysed converter. The elementary averaged models for each switch combination are defined for the relative duty cycles $d_{k}=t_{k} / T_{\text {Seq }}$, as is shown in Eq. (8), where $\boldsymbol{x}$ represents the vectors of the state variables; $\boldsymbol{A}_{k}(t)$ and $\boldsymbol{B}_{k}(t)$ are the state matrix and input matrix for $k$-th switch configuration respectively. The state-space average Eq. (9) for an $\mathrm{MC}$ can be represented by 27 elementary averaged models:

$$
\begin{gathered}
\frac{\mathrm{d} \boldsymbol{x}}{\mathrm{d} t}=\boldsymbol{A}_{k}(t) \boldsymbol{x}+\boldsymbol{B}_{k}(t), \\
\frac{\mathrm{d} \overline{\boldsymbol{x}}}{\mathrm{d} t}=\boldsymbol{A}(d, t) \overline{\boldsymbol{x}}+\boldsymbol{B}(d, t),
\end{gathered}
$$

where:

$$
\sum_{k=1}^{27} d_{k}=1, \quad \boldsymbol{A}(d, t)=\sum_{k=1}^{27} d_{k} \boldsymbol{A}_{k}(d, t), \quad \boldsymbol{B}(d, t)=\sum_{k=1}^{27} d_{k} \boldsymbol{B}_{k}(d, t) .
$$

The coefficient $d_{k}$ is the degree of occurrence of all the possible configurations, and depends on the switch modulation strategy. In an SVM MC only 21 switch configurations are used; 18-active, and 3-zero [22]. Furthermore, the pulse duty factors $d_{k}$ for MCs are a time variable, therefore the presented model is a time-varying one [23]. Using the transformation matrix $\boldsymbol{K}(10)$ we obtain the time-invariant model in the d-q rotating frame. Because the HT output voltage frequency is equal to the supply voltage frequency, then $\boldsymbol{K}$ is defined only for the supply voltage frequency, unlike MC models operating as a frequency converter [22, 23].

$$
\boldsymbol{K}=\left[\begin{array}{ccc}
\boldsymbol{K}_{S} & \ldots & 0 \\
\vdots & \ddots & \vdots \\
0 & \ldots & \boldsymbol{K}_{S}
\end{array}\right], \boldsymbol{K}_{S}=\sqrt{\frac{2}{3}}\left[\begin{array}{ccc}
\cos (\omega t) & \sin (\omega t) & 1 / \sqrt{2} \\
\cos (\omega t-2 \pi / 3) & \sin (\omega t-2 \pi / 3) & 1 / \sqrt{2} \\
\cos (\omega t+2 \pi / 3) & \sin (\omega t+2 \pi / 3) & 1 / \sqrt{2}
\end{array}\right] .
$$

On the assumption of symmetry of HT components, we obtain the final version (11) of the averaged models.

$$
\frac{\mathrm{d} \boldsymbol{Y}}{\mathrm{d} t}=(\boldsymbol{A}-\boldsymbol{\Omega}) \boldsymbol{Y}+\boldsymbol{B},
$$

where:

$$
\overline{\boldsymbol{x}}=\boldsymbol{K} \boldsymbol{Y}, \quad \boldsymbol{\Omega}=\left[\begin{array}{ccc}
\boldsymbol{\Omega}_{S} & \cdots & 0 \\
\vdots & \ddots & \vdots \\
0 & \cdots & \boldsymbol{\Omega}_{S}
\end{array}\right], \quad \boldsymbol{\Omega}_{S}=\left[\begin{array}{ccc}
0 & \omega & 0 \\
-\omega & 0 & 0 \\
0 & 0 & 0
\end{array}\right] .
$$


The solution of Eq. (11) for general conditions and for the steady state is described by (12) and (13) respectively [23], where $\boldsymbol{Y}_{0}$ represents the initial values of state variables, and $\boldsymbol{I}$ is the unit matrix.

$$
\begin{gathered}
\overline{\boldsymbol{x}}=\boldsymbol{K} \boldsymbol{e}^{(A-\boldsymbol{\Omega}) t} \boldsymbol{Y}_{0}+\boldsymbol{K}(\boldsymbol{A}-\boldsymbol{\Omega})^{-1}\left(\boldsymbol{e}^{(A-\boldsymbol{\Omega}) t}-\boldsymbol{I}\right) \boldsymbol{B}, \\
\overline{\boldsymbol{x}}=-\boldsymbol{K}(\boldsymbol{A}-\boldsymbol{\Omega})^{-1} \boldsymbol{B} .
\end{gathered}
$$

The proposed model of the HT should take into account the MC switch modulation strategy. For the SVM of MC switches what should additionally be taken into account is the offset voltage $u_{N n}$ (Fig. 1) [22]. Description of averaged state equations is connected with the calculation of the averaged pulse duty factor for each MC switch $S_{j K}$, which is described by (14).

$$
\left[\begin{array}{l}
\bar{u}_{a} \\
\bar{u}_{b} \\
\bar{u}_{c}
\end{array}\right]=\left[\begin{array}{lll}
d_{a A} & d_{a B} & d_{a C} \\
d_{b A} & d_{b B} & d_{b C} \\
d_{c A} & d_{c B} & d_{c C}
\end{array}\right]\left[\begin{array}{l}
\bar{u}_{A} \\
\overline{u_{B}} \\
\bar{u}_{C}
\end{array}\right]=\overline{\boldsymbol{D}}_{A B C}, \quad\left[\begin{array}{l}
\bar{i}_{A} \\
\bar{i}_{B} \\
\bar{i}_{C}
\end{array}\right]=\left[\begin{array}{lll}
d_{a A} & d_{b A} & d_{c A} \\
d_{a B} & d_{b B} & d_{c B} \\
d_{a C} & d_{b C} & d_{c C}
\end{array}\right]\left[\begin{array}{l}
\bar{i}_{a} \\
\overline{i_{b}} \\
\overline{\bar{i}_{c}}
\end{array}\right]=\boldsymbol{D}^{T} \overline{\boldsymbol{i}}_{a b c} .
$$

The SVM of an MC is described in many publications, e.g., [14, 22], thus switching relations and vector definitions are not shown in this paper. All names of voltage and current vectors, presented later in this article, are compatible with the names presented in the article [22]. In order to determine the voltage $u_{N n}$, consider the switching sequence in the sectors $S_{i}=1$ and $S_{O}=1$. The switch configurations are as follows: $+9,-7,-3,+1$, and matrix switches are switched-on successively by a related time scheme:

$$
\left|d_{\mathrm{I}}\right| \rightarrow\left|d_{\mathrm{II}}\right| \rightarrow\left|d_{\mathrm{III}}\right| \rightarrow\left|d_{\mathrm{IV}}\right|
$$

Then, the transformation matrix $\boldsymbol{D}(14)$ is determined as follows:

$$
\boldsymbol{D}=\boldsymbol{D}_{(+9)}\left|d_{\mathrm{I}}\right|+\boldsymbol{D}_{(-7)}\left|d_{\mathrm{II}}\right|+\boldsymbol{D}_{(-3)}\left|d_{\mathrm{IIII}}\right|+\boldsymbol{D}_{(+1)}\left|d_{\mathrm{IV}}\right|=\left[\begin{array}{ccc}
\left|d_{\mathrm{I}}\right|+\left|d_{\mathrm{II}}\right|+\left|d_{\mathrm{III}}\right|+\left|d_{\mathrm{IV}}\right| & 0 & 0 \\
\left|d_{\mathrm{I}}\right|+\left|d_{\mathrm{II}}\right| & \left|d_{\mathrm{IV}}\right| & \left|d_{\mathrm{III}}\right| \\
0 & \left|d_{\mathrm{II}}\right|+\left|d_{\mathrm{IV}}\right| & \left|d_{\mathrm{I}}\right|+\left|d_{\mathrm{III}}\right|
\end{array}\right]
$$

The same procedure is given for the other sectors with combined $S_{i}$ and $S_{O}$. Offset voltage $u_{N n}$ in the averaged model of the MC with SVM is described by (17) and (18).

$$
\begin{gathered}
\bar{u}_{N}=\frac{\left(\bar{u}_{a}+\bar{u}_{b}+\bar{u}_{c}\right)}{3}, \\
\bar{u}_{N}=\frac{\left(d_{a A}+d_{b A}+d_{c A}\right) \bar{u}_{A}+\left(d_{a B}+d_{b B}+d_{c B}\right) \bar{u}_{B}+\left(d_{a C}+d_{b C}+d_{C}\right) \bar{u}_{C}}{3}=s_{1} \bar{u}_{A}+s_{2} \bar{u}_{B}+s_{3} \bar{u}_{C},
\end{gathered}
$$

where:

$$
s_{1}=\frac{d_{a A}+d_{b A}+d_{c A}}{3}, s_{2}=\frac{d_{a B}+d_{b B}+d_{c B}}{3}, s_{3}=\frac{d_{a C}+d_{b C}+d_{c C}}{3} .
$$


Taking into account expressions (14) - (18), the final state-space averaging Eq. (9) of the HT with the SVM MC are described by (19).

\begin{tabular}{|c|c|c|c|c|c|c|c|c|c|c|c|c|c|c|}
\hline$\frac{\mathrm{d} \bar{i}_{S 1}}{\mathrm{~d} t}$ & $\frac{R_{L F 1}}{L_{F 1}}$ & 0 & 0 & 0 & 0 & 0 & $-\frac{1}{L_{F 1}}$ & 0 & 0 & 0 & 0 & 0 & & \\
\hline $\begin{array}{c}\frac{\mathrm{d} t}{\mathrm{~d} i_{S 2}} \\
\mathrm{~d} t\end{array}$ & 0 & $\frac{R_{L F 2}}{L_{F 2}}$ & 0 & 0 & 0 & 0 & 0 & $-\frac{1}{L_{F 2}}$ & 0 & 0 & 0 & 0 & & $\frac{n_{a} u_{S 1}}{J}$ \\
\hline$\frac{\stackrel{\mathrm{d} t}{\mathrm{~d} i_{S 3}}}{\mathrm{~d} t}$ & 0 & 0 & $\frac{R_{L F 3}}{L_{F 3}}$ & 0 & 0 & 0 & 0 & 0 & $-\frac{1}{L_{F 3}}$ & 0 & 0 & 0 & $i_{S 1}$ & $\begin{array}{c}L_{F 1} \\
n_{a} u_{S 2} \\
L_{F 2}\end{array}$ \\
\hline$\frac{\mathrm{d} i_{L S 1}}{\mathrm{~d} t}$ & 0 & 0 & 0 & $\frac{R_{L L 1}}{L_{L 1}}$ & 0 & 0 & $\frac{d_{a A}-s_{1}}{L_{L 1}}$ & $\frac{d_{a B}-s_{2}}{L_{L 1}}$ & $\frac{d_{a C}-s_{3}}{L_{L 1}}$ & $\frac{-1}{L_{L 1}}$ & 0 & 0 & $\begin{array}{l}i_{S 2} \\
\bar{i}_{S 3}\end{array}$ & $\frac{n_{a 2}^{L_{F 3}}}{L_{F 3}}$ \\
\hline$\frac{\mathrm{d} i_{L S 2}}{\mathrm{~d} t}$ & 0 & 0 & 0 & 0 & $-\frac{R_{L L 2}}{L_{L 2}}$ & 0 & $\frac{d_{b A}-s_{1}}{L_{L 2}}$ & $\frac{d_{b B}-s_{2}}{L_{L 2}}$ & $\frac{d_{b C}-s_{3}}{L_{L 2}}$ & 0 & $\frac{-1}{L_{L 2}}$ & 0 & $\begin{array}{l}i_{L L L} \\
i_{-}\end{array}$ & 0 \\
\hline$\underline{\mathrm{d} \bar{i}_{L S 3}}$ & 0 & 0 & 0 & 0 & 0 & $R_{L L 3}$ & $\underline{d_{c A}-s_{1}}$ & $\underline{d_{c B}-s_{2}}$ & $\underline{d_{c C}-s_{3}}$ & 0 & 0 & -1 & ${ }^{l_{L L 2}}$ & 0 \\
\hline$\stackrel{\mathrm{d} t}{-}$ & & & & & & $L_{L 3}$ & $L_{L 3}$ & $L_{L 3}$ & $L_{L 3}$ & & & $L_{L 3}$ & $\begin{array}{l}i_{L L 3} \\
-\end{array}$ & 0 \\
\hline$\frac{\mathrm{d} u_{C F}}{\mathrm{~d} t}$ & $\frac{1}{C}$ & 0 & 0 & $\frac{a_{a A}}{C}$ & $-\frac{a_{b A}}{C}$ & $-\frac{d_{c A}}{C}$ & 0 & 0 & 0 & 0 & 0 & 0 & $u_{C}{ }_{C F}$ & 0 \\
\hline$\stackrel{\mathrm{d} t}{\mathrm{~d} u_{C F 2}}$ & $C_{F 1}$ & 1 & & $\begin{array}{l}C_{F 1} \\
d_{a B}\end{array}$ & $\begin{array}{l}C_{F 1} \\
d_{b B}\end{array}$ & $\begin{array}{l}C_{F 1} \\
d_{c B}\end{array}$ & & & & & & & $\bar{u}_{C F 2}$ & 0 \\
\hline$\frac{\mathrm{d} t}{\mathrm{~d} u_{C F 3}}$ & 0 & $\overline{C_{F 2}}$ & 0 & $\overline{C_{F 2}}$ & $\overline{C_{F 2}}$ & $\overline{C_{F 2}}$ & 0 & 0 & 0 & 0 & 0 & 0 & $u_{C F 3}$ & $\begin{array}{c}0 \\
-n_{b} u_{S 1} \\
\end{array}$ \\
\hline$\frac{\mathrm{d} t}{\mathrm{~d} u_{L 1}}$ & 0 & 0 & $\frac{1}{C_{F 3}}$ & $-\frac{a_{a C}}{C_{F 3}}$ & $-\frac{a_{b C}}{C_{F 3}}$ & $-\frac{a_{c C}}{C_{F 3}}$ & 0 & 0 & 0 & 0 & 0 & 0 & $\bar{u}_{L 2}$ & $\begin{array}{l}\overline{R_{L 1} C_{L 1}} \\
-n_{b} u_{S 2}\end{array}$ \\
\hline$\overline{\mathrm{d} t}$ & 0 & 0 & 0 & $\frac{1}{C_{L 1}}$ & 0 & 0 & 0 & 0 & 0 & $\frac{-1}{R_{L 1} C_{L 1}}$ & & 0 & $u_{L 3}$ & $\begin{array}{l}\overline{R_{L 2} C_{L 2}} \\
-n_{b} u_{S 3}\end{array}$ \\
\hline $\begin{array}{c}\overline{\mathrm{d} t} t \\
\mathrm{~d} u_{L 3}\end{array}$ & 0 & 0 & 0 & 0 & $\frac{1}{C_{L 2}}$ & 0 & 0 & 0 & 0 & 0 & $\frac{-1}{R_{L 2} C_{L 2}}$ & 0 & & $\overline{R_{L 3} C_{L 3}}$ \\
\hline$\overline{\mathrm{d} t}$ & 0 & 0 & 0 & 0 & 0 & $\overline{C_{L 3}}$ & 0 & 0 & 0 & 0 & 0 & $R_{L 3} C_{L 3}$ & & \\
\hline
\end{tabular}

\section{Validation of HT averaged model}

Static characteristics obtained from the mathematical model (19) are presented in Fig. 3. The voltage gain $K_{U}$ as a function of the setting of the MC output voltage phase shift $\varphi_{L}$ for different MC voltage gains $q$ is show in Fig. 3a. The adjustment range of the amplitude of the HT output voltage is shown with 3D characteristics in Fig. 3b. As can be seen from these characteristics, it is possible to obtain the amplitude of output voltage $U_{L}$ equal to the amplitude of source voltage $U_{S}$, with and without a phase shift between source and output voltages. It should also be noted that it is possible to obtain an output voltage equal to zero for $q=0.5$ and $\varphi_{L}$ approximately equal to $220^{\circ}$. Then, the matrix converter output voltage is shifted by $180^{\circ}$ relative to the voltage $u_{b}$ of the transformer secondary winding and has the same amplitude. An output voltage in antiphase to the mains voltage it also achieved.

The general function of the HT is compensation of AC voltage sags and overvoltage. Thus, the HT works as a power transformer with a dynamic voltage restorer (DVR) function. Examples of AC voltage stabilization during a $70 \%$ source voltage sag and $120 \%$ source overvoltage, using the HT with the MC are presented in Fig. 4a. As can be seen in the presented time waveforms (Fig. 4a), the moments of start and end of the voltage sag or overvoltage are almost imperceptible, because both amplitude and phase shift $\delta$ are compensated. A large number of voltage sags in the power network are single-phase or unbalanced sags, resulting from a single-phase short circuit to ground, a two-phase short circuit or a two-phase short circuit to ground $[3,4]$. The analysis of this type of compensation requires a complex form of 
power converter switch modulation strategy. Fig. $4 \mathrm{~b}$ shows the simulation results of unbalanced sag compensation using the proposed HT. Before and during the disturbance the output voltages have constant amplitude, but there can be seen some deformation in the shape of the output voltages - low harmonic distortion [24]. In addition, simulation time waveforms of voltages in the system for unbalanced and nonlinear loads are shown in Fig. 6. For unbalanced load (Fig. 6a), the HT allows compensation of voltage sag and overvoltage with a similar accuracy as for a symmetrical load (Fig. 5a). It should be noted that, for non-linear loads there is considerable distortion of the output voltage (Fig. 6). All the presented simulation waveforms were obtained in an open control loop, since the main aim of this article was to describe the basic properties of the HT. Using a closed control loop a reduction in output voltage distortion is to be expected.

All theoretical and simulation studies have been done using the following parameters: source voltage $230 \mathrm{~V} / 50 \mathrm{~Hz}$, input and output filter inductance and their resistances $L_{F}=L_{L}$ $=1 \mathrm{mH} / 0.01 \Omega$, input and output filter capacitance $C_{F}=C_{L}=20 \mu \mathrm{F}$, switching frequency $f_{\text {Seq }}=5 \mathrm{kHz}$ and simulation step $T_{p}=1 \mu \mathrm{s}$. It should be noted that in the simulation investigation idealized bidirectional switches are used. None of the commutation strategies of bidirectional switches that are commonly used in MCs $([14,25])$ are used in the simulation model. Different commutation strategies in an $\mathrm{MC}$ are used to provide the correct current switching pattern for bidirectional switches and to eliminate distortion in the voltage and current waveforms, which occurs when the "dead time" between two control signals is used [25].

The proposed solution of an HT with an MC has some drawbacks, related to the connections of the HT to the same system in which there is a grid fault. Because the system has no energy storage element, the HT needs a minimum network voltage to work properly and it may not be able to compensate very deep sags [16].

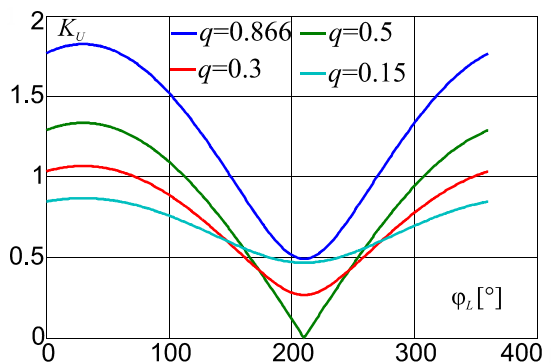

(a)

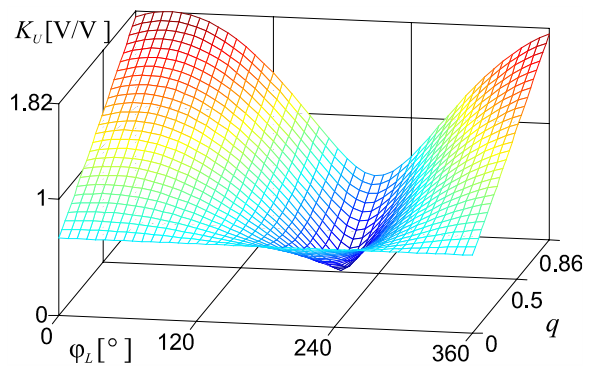

(b)

Fig. 3. Voltage gain $K_{U}$ of HT as a function of: output voltage phase shift $\varphi_{L}$ for different MC setting voltage gains $q$ (a), output voltage phase shift $\varphi_{L}$ and $\mathrm{MC}$ voltage gain $q(\mathrm{~b})$

Fig. 6 compares the simulation results obtained in Matlab Simulink software for the presented HT and the resulting averaged models in transient states. To provide a meaningful comparison of the simulation model and the averaged model, the corresponding time waveforms of output voltage $u_{L 2}$, obtained from both models are juxtaposed in one graph. The corresponding results from both models agree very well with and validate the accuracy of the averaged 
model versus the simulation model. Both results demonstrate good correlation, which confirms the usefulness of the analytical averaged method used in the analysis of the HT properties.

a)

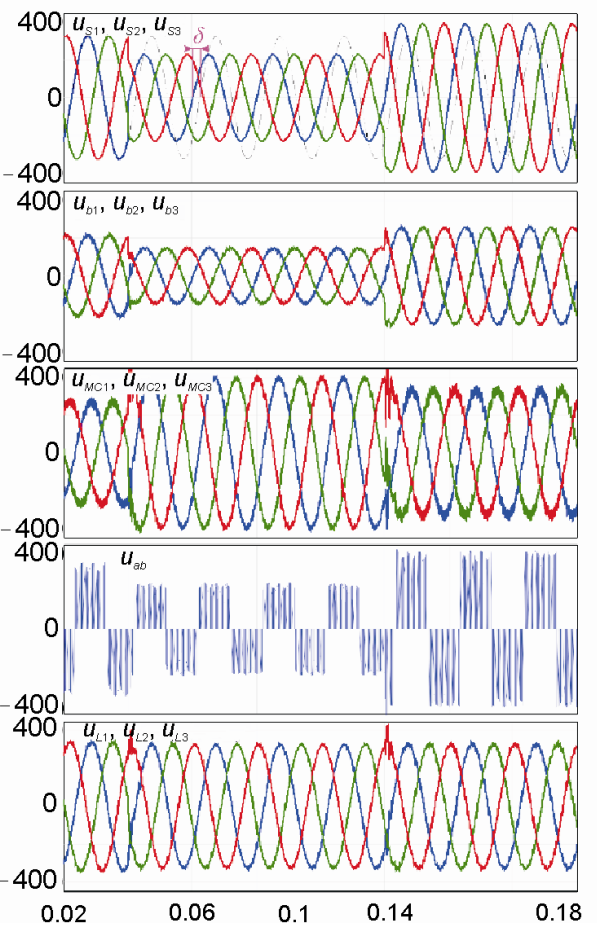

b)

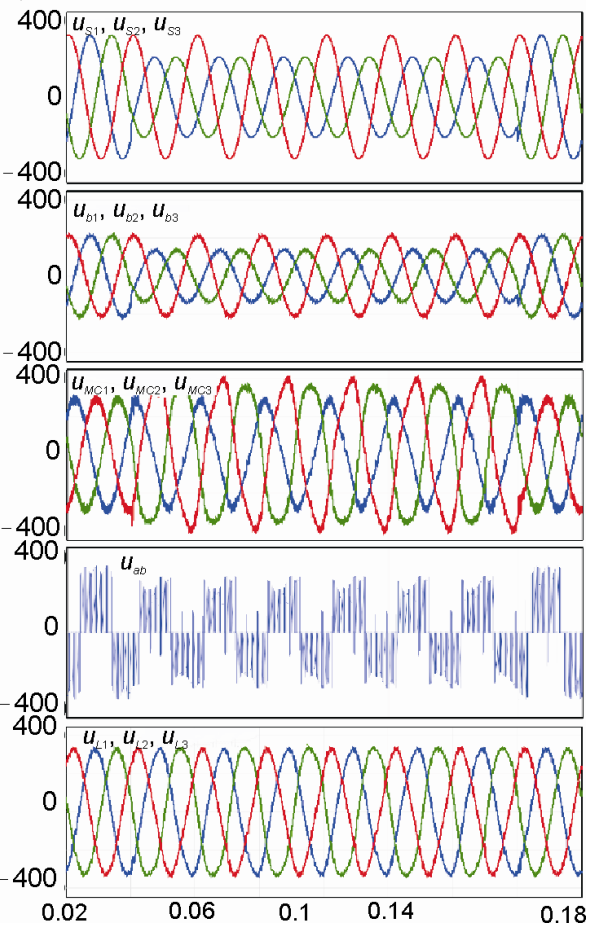

Fig. 4. Simulation results of HT with $\mathrm{MC}$ as a voltage compensator: voltage time waveforms during balanced $70 \%$ source voltage sag, and $120 \%$ source overvoltage (a); voltage time waveforms during $65 \%$ unbalanced source voltage sag, which are the result of two-phase short circuit to ground (b)

\section{Conclusions}

This paper has presented a study of the properties and a mathematical averaged model of a three-phase hybrid transformer with a matrix converter. The electrical circuit scheme and its operational conditions have been described. Furthermore, a detailed mathematical model based on average equations for an MC with SVM has also been presented. The proposed HT is able to compensate voltage sags and overvoltage, as shown in the simulation investigation. Compensation of both amplitude and resulting voltage phase shift, even for asymmetrical deformations, is possible in the analyzed system.

It should be emphasized that such a system may already be easily implemented to protect consumers in a power range equal to a few kVA. The next step of the research will focus on experimental applications in closed loop control, and define a compensation range of voltage sag and overvoltage. 
a)

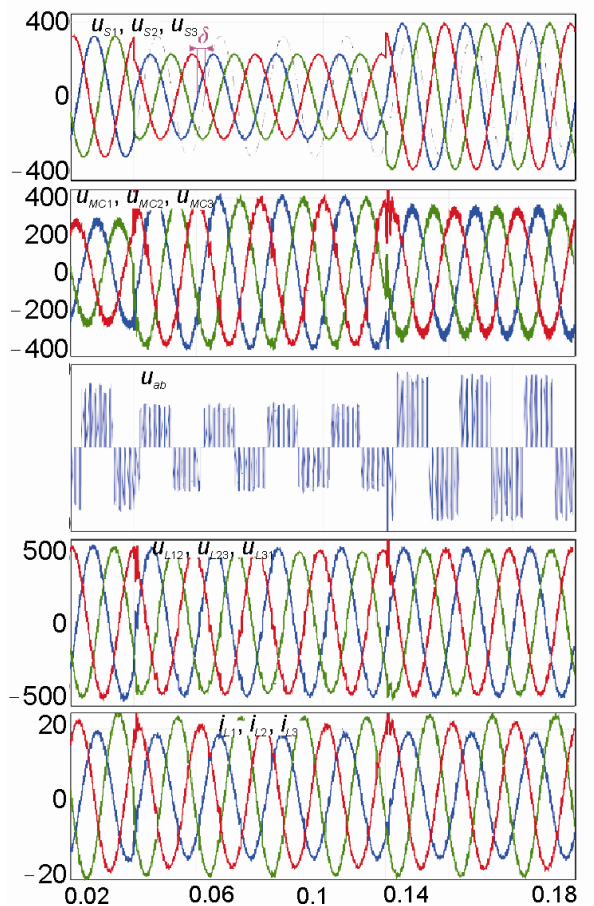

b)

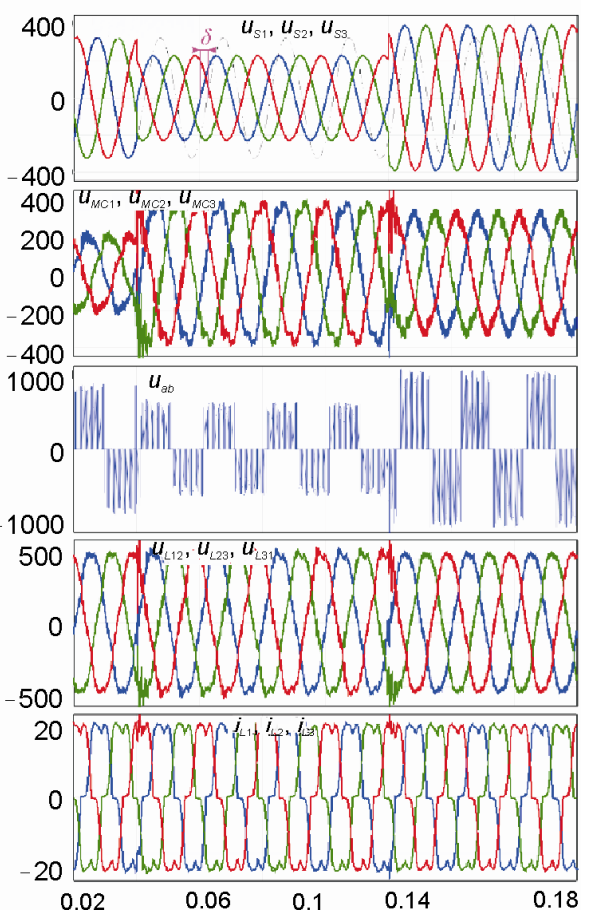

Fig. 5. Simulation results of HT with MC as a voltage compensator during balanced $70 \%$ source voltage sag, and $120 \%$ source overvoltage, for unbalanced load (a), for nonlinear load (b)

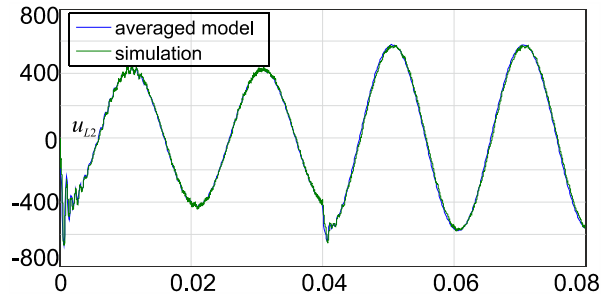

Fig. 6. Comparison of HT simulation and average models - time waveforms of output voltage $u_{L 2}$, obtained during simulation process and average modelling

\section{Acknowledgements}

The research was carried out within the scope of the project funded by the "National Science Centre, Poland" under the reference number 2015/19/D/ST7/01371.

\section{References}

[1] EN 50160, Voltage characteristics of electricity supplied by public distribution systems (2007).

[2] IEEE Standard 1159, Recommended practice for monitoring electric power quality (2009).

[3] Mahela O., Shaik A.G., Gupta N., A critical review of detection and classification of power quality events, Renewable \& Sustainable Energy Reviews, vol. 41, pp. 495-505 (2015).

[4] Jurado F., Saenz J.R., Detecting transient voltage stability and voltage sag, COMPEL, The International Journal for Computation and Mathematics in Electrical and Electronic Engineering, vol. 23 , no. 2 , pp. 392-409 (2004). 
[5] Ekström R., Thomas K., Leijon M., Fast solid-state on-load tap change using two currentcontrolled voltage-source inverters, IET Power Electronics, vol. 7, no. 10, pp. 2610-2617 (2014).

[6] Choi J-H., Moon S-I., The dead band control of LTC transformer at distribution substation, IEEE Transactions on Power Systems, vol. 24, no. 1, pp. 319-326 (2009).

[7] Nielsen J.G., Blaabjerg F., A Detailed comparison of system topologies for dynamic voltage restorers, IEEE Transactions on Industrial Applications, vol. 41, no. 5, pp. 1272-1280 (2005)

[8] Torres A.P., Roncero-Sánchez P., del Toro García X., Batlle V.F., Generalized proportional-integral controller for dynamic voltage restorers, COMPEL, The International Journal for Computation and Mathematics in Electrical and Electronic Engineering, vol. 31, no. 6, pp.1964-1984 (2012).

[9] Benysek G., Improvement in the quality of delivery of electrical energy using PE systems, Springer (2007).

[10] Kaniewski J. Szcześniak P., Jarnut M., Benysek G., Hybrid Voltage Sag/Swell Compensators: A Review of Hybrid AC/AC Converters, IEEE Industrial Electronics Magazine, vol. 9, no. 4, pp. 37-48 (2015).

[11] Kaniewski J., Fedyczak Z., Benysek G., AC voltage sag/swell compensator based on three-phase hybrid transformer with buck-boost matrix-reactance chopper, IEEE Transactions on Industrial Electronics, vol. 61, no. 8, pp. 3835-3846 (2014).

[12] Szcześniak P., Kaniweski J., Hybrid transformer with matrix converter, IEEE Transactions on Power Delivery, vol. 31, no. 3, pp. 1388-1396 (2016).

[13] Burkard J., Biela J., Evaluation of topologies and optimal design of a hybrid distribution transformer, European Conference on Power Electronics and Applications, EPE'15 ECCE Europe, Geneva, Switzerland, pp. 1-10 (2015).

[14] Rodriguez J., Rivera M., Kolar J.W., Wheeler P.W., A review of control and modulation methods for matrix converters, IEEE Transactions on Industrial Electronics, vol. 59, no. 1, pp. 58-70 (2012).

[15] Szcześniak P., Kaniewski J., Jarnut M., AC-AC power electronic converters without DC energy storage: a review, Energy Conversion and Management, vol. 92, pp. 483-497 (2015).

[16] Szcześniak P., Kaniewski J., Power electronics converters without DC energy storage in the future electrical power network, Electric Power Systems Research, vol. 129, pp. 194-207 (2015).

[17] Kaniewski J., Fedyczak Z. Szcześniak P., Three-phase hybrid transformer using matrix-chopper as an interface between two AC voltage sources, Archives of Electrical Engineering, vol. 63, no. 2, pp. 197-210 (2014).

[18] Montero-Hernandez O.C., Enjeti P.N., Application of a boost AC/AC converter to compensate for voltage sags in electric power distribution systems, Power Electronics Specialists Conference PESC 2000, Galway, Ireland, pp. 470-475 (2000).

[19] Aeoliza E.C., Enjeti N.P., Moran L.A., Montero-Hernandez O.C., Kim S., Analysis and design of a novel voltage sag compensator for critical loads in electrical power distribution systems, IEEE Transactions on Industrial Applications, vol. 39, no. 4, pp. 1143-1150 (2003).

[20] Choi N., Li Y., Modeling and analysis of ac line conditioner based on three-phase PWM Ćuk ac-ac converter, Annual Conference of the IEEE Industrial Electronics Society, IECON'04, Busan, Korea, pp. 1646-1651 (2004).

[21] Minh-Khai N., Young-Cheol L., Joon-Ho C., Single-phase Z-source based voltage sag/swell compensator, Applied Power Electronics Conference and Exposition, APEC'13, Long Beach, USA, pp. 3138-3142 (2013).

[22] Szcześniak P., A static and dynamic model of a space vector modulated matrix-reactance frequency converter, Electric Power Systems Research, vol. 108, pp. 82-92 (2014).

[23] Korotyeyev. I,Y., Fedyczak Z., Steady and transient states modelling methods of matrix-reactance frequency converter with buck-boost topology, COMPEL, The International Journal for Computation and Mathematics in Electrical and Electronic, vol. 28, no. 3, pp. 626-638 (2009).

[24] Lozano J.M., Ramirez J.M., Correa R.E., A novel dynamic voltage restorer based on matrix converters, Modern Electric Power Systems, MEPS'10, Wroclaw, Poland, pp. 1-7 (2010).

[25] Wheeler P.W., Rodriguez J, Clare J.C., Empringham L., Weinstejn A., Matrix converters: a technology review, IEEE Transactions on Industrial Electronics, vol. 49, no. 2, pp.276-288 (2002). 